\title{
2D turbulence imaging in DIII-D via beam emission spectroscopy
}

\author{
C. Fenzi, R. J. Fonck, M. Jakubowski, and G. R. Mc Kee \\ University of Wisconsin, Madison, Wisconsin 53706
}

(Presented on 21 June 2000)

Two-dimensional measurements of density fluctuations have been performed in DIII-D using the beam emission spectroscopy diagnostic. The 32 spatial channels are arranged to image a 5 $\times 6 \mathrm{~cm}^{2}$ (radial $\times$ poloidal) region in the plasma cross section, at a nominal $1 \mathrm{~cm}$ spatial resolution and separation. The typical decorrelation time, poloidal and radial correlation lengths, as well as a time-averaged flow field plot are obtained from spatial and temporal correlation analyses. A biorthogonal decomposition algorithm is applied to expand the data set into a set of modes that are orthogonal in time and in space, thus providing a simultaneous analysis of the space and time dependencies of fluctuation data. (C) 2001 American Institute of Physics.

[DOI: $10.1063 / 1.1321739$ ]

\section{INTRODUCTION}

Turbulence in a toroidally confined tokamak plasma is fundamentally two-dimensional (2D) in nature. Hence, fully 2D measurements are needed for obtaining the relevant $S\left(k_{r}, k_{\theta}\right)$ wave number spectrum and a realistic representation of the turbulence structures for comparison to theory, to provide time-averaged and possibly time-resolved visualization of the turbulent eddy structures, obtain high spatial resolution fluctuation velocity and velocity shear measurements, and ultimately characterize the turbulence growth and nonlinear energy transfer rates for more direct comparison to nonlinear theory. The 2D measurements have been obtained with Langmuir probes array in a relatively cold plasma. ${ }^{1}$ In DIII-D, the beam emission spectroscopy (BES) diagnostic allows for such measurements in hot plasmas, and provides the first direct visualization of 2D turbulent structures.

To advance beyond applications of conventional twopoint spatial analysis and straightforward visualization of the turbulence, there is a need to define techniques to analyze time- and space-resolved signals without involvement of long-time averages of moments of data. Usual spectral analysis techniques suffer from a lack of time and/or space resolution, and the approach to fully $2 \mathrm{D}$ signal analysis remains undetermined.

The biorthogonal decomposition technique (BD) has been suggested as a method allowing for a simultaneous analysis of temporal and spatial dynamics of multidimensional data. ${ }^{2-5}$ It has been successfully applied to $1 \mathrm{D}$ Langmuir probe data from the Axially Symmetric Divertor Experiment (ASDEX) and ADITYA tokamaks, as well on 2D simulated data. ${ }^{6}$ By expanding the data into a set of modes that are orthogonal in time and in space, identification and extraction of coherent structures in the signal are possible. Moreover, such a decomposition may aid the investigation of nonlinear energy cascades between spatial modes.

In the present article, we present 2D images of turbulence in a tokamak plasma and extend the BD technique to fully 2D time- and space-resolved experimental BES data. The remainder of this article is as follows: in Sec. II we describe the BES experimental setup for 2D fluctuation measurements, and 2D images of turbulence are presented in Sec. III. Details of the BD technique are introduced in Sec. IV, and preliminary results are presented. Finally, Sec. V contains some conclusions and prospects.

\section{THE BES DIAGNOSTIC SETUP FOR 2D MEASUREMENTS}

The beam emission spectroscopy diagnostic installed on DIII-D measures the light emitted from an injected highpower $\mathrm{D}^{0}$ neutral beam, and provides measurements of spatially localized long wavelength density fluctuations. The diagnostic has been described in detail elsewhere, ${ }^{7}$ and only details necessary for the understanding of the present work are given here. The BES system has 32 spatial channels which can be deployed in both radial and poloidal directions. The $1 \mathrm{~cm}$ radial and vertical resolution allows for observation of long wavelength modes with wavenumber $k$ $<3 \mathrm{~cm}^{-1}$, which are typically associated with anomalous transport in the edge and core plasma regions. The $2 \mathrm{D}$ motorized fiber mounting array can be scanned radially to observe different spatial regions of the plasma on a shot-to-shot basis. Finally, the signals are digitized at $1 \mathrm{MHz}$ and the use of cryogenically cooled amplifier circuits provides photonnoise-limited current detection.

For the measurements discussed here, spatial channels are arranged to image a $5 \times 6 \mathrm{~cm}^{2}($ radial $\times$ poloidal) region in a plasma poloidal cross section, at a nominal $1 \mathrm{~cm}$ spatial resolution and spatial separation. Data have been acquired during L-mode plasmas in upper single null configuration (ion $\nabla B$ drift away from the $\mathrm{X}$-point) and the edge-imaged region covers $0.87 \leqslant r / a \leqslant 1$ near the outer plasma midplane. 

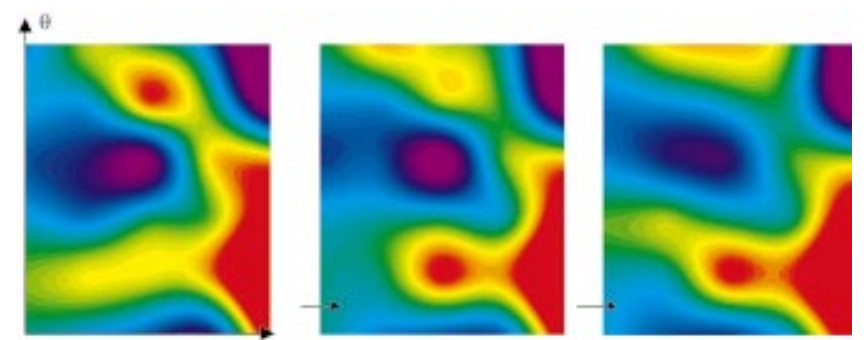

$0.87<n a<1$

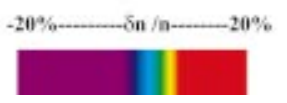

FIG. 1. (Color) Evolutions of 2D density fluctuation contours in an L-mode plasma. The time delay between each frame is $1 \mu \mathrm{s}$.

\section{VISUALIZATION OF DENSITY FLUCTUATION STRUCTURES}

This 2D measurement configuration provides for the first time a direct visualization of 2D structures in turbulence in the closed flux surfaces of a hot tokamak plasma. Three temporal frames of imaged density, separated by $1 \mu$ s in time, are presented in Fig. 1. The data have been frequency filtered over the region of broadband fluctuations. To improve the visualization, the $5 \times 6$ array of spatial grid points has been expanded to a $50 \times 60$ array using a $2 \mathrm{D}$ spline fit, then linearly interpolated to a $250 \times 300$ pixel image, as presented. Large scale, transient, and coherent structures (localized in time and in space) are observed convecting upward through the observation domain at several kilometers per second, as determined from time-delay correlation analysis. Long-lived structures (persisting for several turbulent decorrelation times) as well as cascadelike phenomenon from large to small spatial scale are observed. Figure 2 illustrates the 2D time-averaged turbulent flow-field plot inferred from these analyzed data. The fluctuation group velocity decreases towards the plasma edge, and reverses near the separatrix $(r / a=1)$. Spatial and temporal correlation analyses indicate that the typical fluctuation correlation times are 7-24 $\mu \mathrm{s}$.

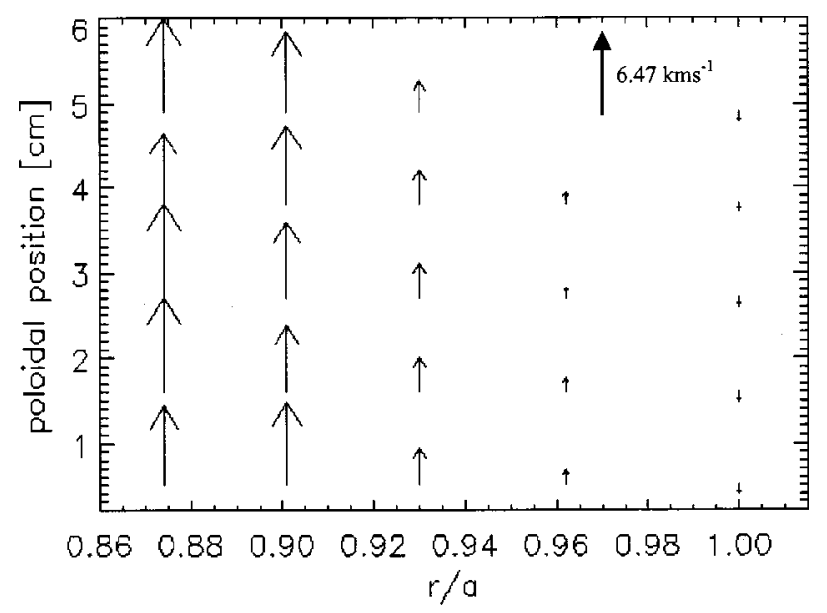

FIG. 2. Flow-field plot of BES data during an L-mode upper single null plasma, the imaged plasma region being $0.87<r / a<1$. Group velocities are inferred from two points cross-correlation analyses between two poloidal adjacent channels, the poloidal convection of the fluctuations supposedly being dominated by the $E \times B$ flow.
The radial and poloidal cross-correlation lengths are of the order of 2.5 and $3.35 \mathrm{~cm}$, respectively, showing an asymmetry in the averaged eddy structure.

\section{SEARCH FOR COHERENT STRUCTURES IN 2D BES DATA}

It is notable that the $2 \mathrm{D}$ images provide quantitative information on the turbulence only through conventional twopoint correlation analysis. Higher-order analysis, such as studies of nonlinear mode coupling in 2D data, ${ }^{8}$ provides measurement of the coupling and power flow between different spatial wavelengths. To complement such analysis, there is interest in exploring means of correlating spatial and temporal properties of the turbulence, and search for longlived coherent structures.

The conventional two-point time-averaged correlation analysis does not allow the identification of such long-lived coherent structures in the signal, if they exist. In fact, the statistical properties mentioned above are averaged over the observed $k$-spectrum, so that they could be related to the most unstable mode or to the existence of coherent structures.

In contrast, the biorthogonal decomposition technique (BD) allows for such identification and characterization of coherent structures. This method has been already applied to 1D Langmuir probe measurements in the edge region of the ASDEX and ADITYA tokamaks. ${ }^{6}$ Basically, the BD technique realizes the projection of the data in an orthogonal basis in space and time, then provides the simultaneous analysis of the space and time dependencies of the fluctuation data. We extend here the method to the full 2D timeand space-resolved BES data as a first step in developing a more quantitative analysis of 2D turbulence data.

The BD technique approach can be illustrated considering a 2D scalar spatio-temporal signal $S(r, \theta, t)$ measured at $N$ different spatial locations $(r, \theta)$ with $M$ temporal frames. The data are assembled into an $N \times M$ array $S(z, t)$ where the columns contain the spatial information of the signal. Using a singular value decomposition algorithm, the $\mathrm{BD}$ technique expands the discrete data $S_{i j}=S\left(Z_{j}, t_{i}\right)$ into a unique set of modes that are orthogonal in time and in space so that $S_{i j}$ $=\sum_{n=1}^{\min (N, M)} \lambda_{n} C_{n}\left(t_{i}\right) T_{n}\left(z_{j}\right)$ with the one-to-one correspondence $S T_{n}=\lambda_{n} C_{n}$. Here, $\lambda_{n}, C_{n}$, and $T_{n}$ are, respectively, the weight component, the temporal component, and the spatial component [to be reshaped so that $T_{n}(z)=T_{n}(r, \theta)$ ] eigensolutions of, respectively, the original data matrix $\mathbf{S}$, the twopoint temporal, and the two-point spatial cross-correlation matrices.

The latter property is very important here. It means that the higher are the weight components, the stronger are the time and/or space correlations. The components that are associated with large weights represent highly correlated structures and reflect dominant features of the signal. Totally uncorrelated signals (like random noise) will lead to components of equal weight.

It is also worth noting that the BD technique is not equivalent to a Fourier analysis where the basis functions are prespecified. Here, the basis functions are calculated from 


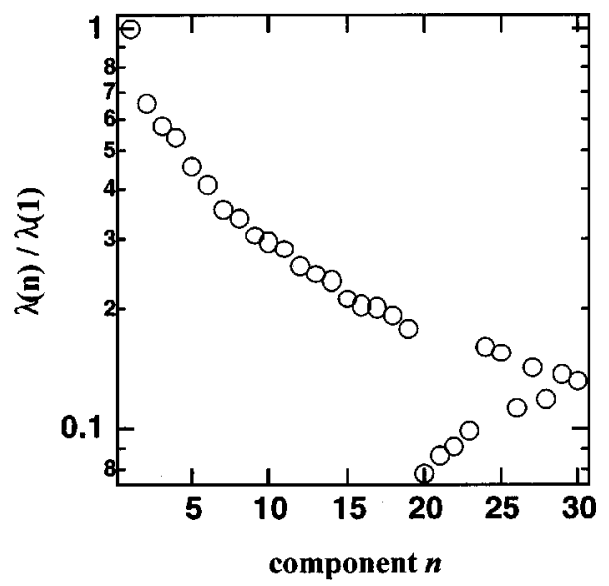

FIG. 3. Log distribution plot of the 30 weight components $\lambda_{n}$. The distribution has been normalized to the value of $\lambda_{1}$.

the data themselves. It turns out that this data-adaptive property of the BD technique allows us to directly isolate and identify structures (part of the signal that is correlated in time and in space) in the signal without any a priori knowledge about their shapes. Finally, the one-to-one correspondence between the spatial and the temporal BD components guaranties the signal to be fully reconstructed from its components. ${ }^{4}$

Figure 3 illustrates the weight component distribution for the analyzed data. The steepness of this distribution is an indication of redundancy in the data, and just a few modes are necessary to describe the whole data set. Indeed, the first 14 modes (associated to the first fourteen larger $\lambda_{n}$ ) represent together over $90 \%$ of the signal power, and the break in the slope illustrates the transition to the noisy regime.

An example of the derived temporal and spatial components are represented in Fig. 4, showing the first three biorthogonal temporal and spatial components associated with the first three weight components (which together represent over $50 \%$ of the signal power). As the mode number increases, the mode spatial structure tends to oscillate and the associated temporal component exhibits a higher frequency distribution, the latter being ultimately increasingly affected by noise. Also, the shape of the spatial modes changes minimally when applying the BD decomposition to different time windows. Moreover, the frequency power spectral density of the temporal modes is narrow for the lower-order modes, whereas the higher-order ones have a broader spectrum. Low-frequency components are associated with large structures, whereas higher frequency components are associated with smaller ones, consistent with the picture of eddies of various sizes convecting in a flow field.

Finally, Fig. 5 illustrates the data after subtraction of the first 14 dominant components. A comparison of Figs. 5 and 1 clearly shows that the large structures have completely disappeared, i.e., the dominant components capture the large structures in the signal. It stresses the ability of the method to separate different scales (spatial or temporal instead of just frequencies or wavelengths) as previously mentioned. ${ }^{6}$
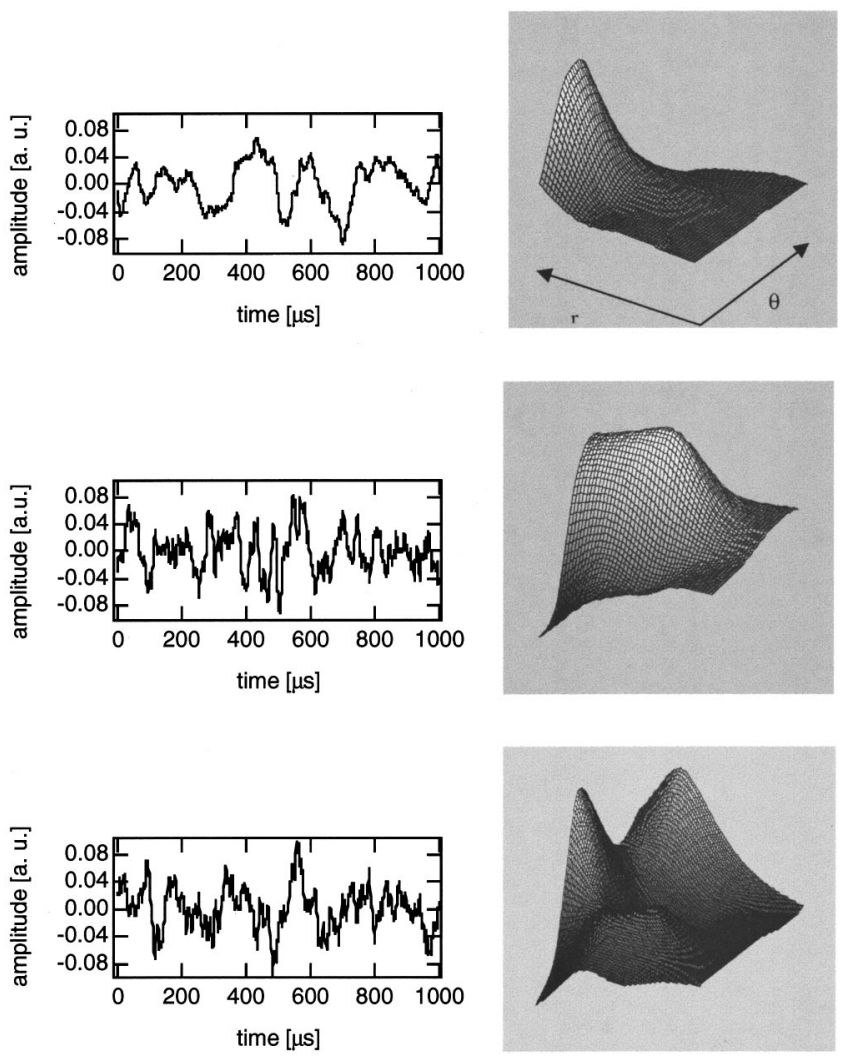

FIG. 4. The three first dominant biorthogonal modes (left: temporal component, right: spatial component) associated to the BES data (1 ms i.e., 1000 frames analyzed). Data have been splined and interpolated to improve the visualization.

\section{DISCUSSION AND PROSPECTS}

The BES diagnostic on DIII-D provides 2D measurements of density fluctuations and the first visualization of turbulent structures in a hot tokamak plasma. The biorthogonal decomposition technique has been extended to 2D experimental fluctuation data, allowing for the simultaneous analysis of the temporal and spatial dynamics. This technique appears to be a powerful tool to isolate and characterize structures in the turbulent signal. The data set decomposition in modes that are orthogonal in time and in space should facilitate the investigation of nonlinear coupling and energy transfer between modes. In fact, the BD technique provides crucial information about the coupling between the
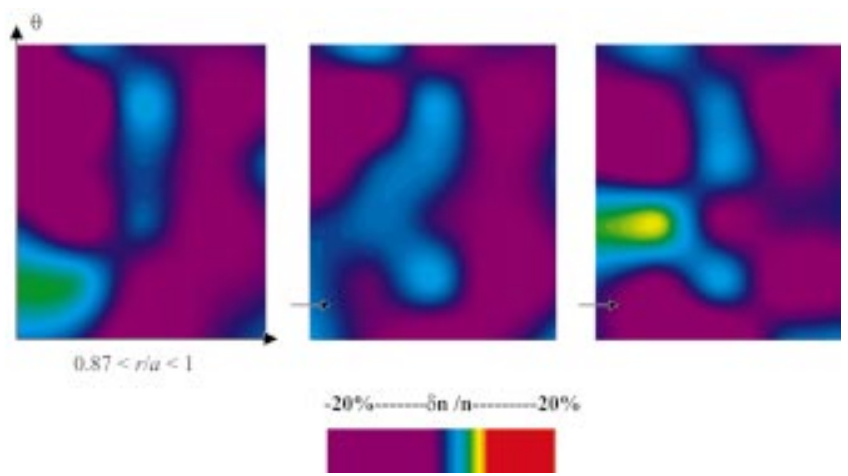

FIG. 5. (Color) Reconstruction of the BES data after subtraction of the first 14 biorthogonal modes (compare to Fig. 1). 
spatial and temporal mode behavior. That is complementary to a nonlinear analysis which estimates energy transfer between spatial modes. In complement to the BD analysis, a nonlinear algorithm involving the bispectral analysis technique $^{8,9}$ is being adapted to $2 \mathrm{D}$ BES data in order to determine linear growth/damping rate, nonlinear coupling between turbulent modes in the plasma as well as nonlinear energy transfer rate between modes.

\section{ACKNOWLEDGMENTS}

This work was supported by U.S. Department of Energy Grant No. DE-FG02-89ER53296 and by DGCID (Direction
Générale de la Coopération Internationale et du Développement) of France under the scientific program Lavoisier.

${ }^{1}$ S. J. Zweben and R. W. Gould, Nucl. Fusion 35, 171 (1985).

${ }^{2}$ N. Aubry, R. Guyonnet, and R. Lima, J. Stat. Phys. 64, 693 (1991).

${ }^{3}$ C. Nardone, Plasma Phys. Controlled Fusion 34, 1447 (1992).

${ }^{4}$ T. Dudok de Wit et al., Phys. Plasmas 1, 3288 (1994).

${ }^{5}$ T. Dudok de Wit, Plasma Phys. Controlled Fusion 37, 117 (1995).

${ }^{6}$ S. Benkadda et al., Phys. Rev. Lett. 73, 3403 (1994).

${ }^{7}$ G. McKee et al., Rev. Sci. Instrum. 70, 913 (1999).

${ }^{8}$ J. S. Kim et al., Phys. Plasmas 3, 3998 (1996).

${ }^{9}$ J. S. Kim et al., Phys. Rev. Lett. 79, 841 (1997) 